\title{
NEFRECTOMÍA LAPAROSCÓPICA DE DONANTE VIVO: EXPERIENCIA DE 75 CASOS CONSECUTIVOS*
}

\author{
Drs. Jorge Aguiló M. ${ }^{1}$, Carlos Matus F. ${ }^{1}$, Luis Leiva L. ${ }^{1}$, Pablo Pérez C. ${ }^{1}$, Felipe Castillo H. ${ }^{1}$, \\ Jorge Vergara M. ${ }^{1}$, Jorge Gaete V. ${ }^{1}$, Ints. Pablo Alarcón T. ${ }^{2}$, Daniel Donoso P. ${ }^{2}$
}

1 Departamento de Cirugía Sur, Universidad de Chile, Hospital Barros Luco-Trudeau (HBLT).

2 Internos de Medicina, Universidad de Santiago.

Santiago, Chile.

\begin{abstract}
Live donor laparoscopic nephrectomy. Experience in 75 cases

Background: Live Donor Laparoscopic Nephrectomy (LDLN) has substantial benefits when compared with open nephrectomy such as shorter hospital stay, prompt return to work, less post-operative pain, better cosmetic results, less blood loss and less surgical wound infections. It is the mode of choice for safely harvesting a kidney for organ transplantation. Aim: To describe the surgical results of LDLN in a pioneer renal transplant center in Chile. Material and Methods: Review of clinical records of 75 subjects aged 27 to 60 years (37 males) subjected to a LDLN in a public hospital between 1998 and 2013. Information about clinical and surgical data and perioperative complications was retrieved. Results: No subject died. All kidneys were satisfactorily implanted in their receptors. The mean operative time was 116 minutes. Mean hospital stay was 1.6 days, warm ischemia time was 6.8 minutes, and cold ischemia time was 31.5 minutes. Operative adverse events occurred in $8 \%$. The conversion and reoperation rates were 4 and $1.3 \%$, respectively. Among receptors, $1.5 \%$ evolved with Acute Tubular Necrosis and 2.2\% required graft excision. Conclusions: LDLN is a safe and pioneering surgical technique in Chile. Its results are satisfactory and comparable to those obtained with classic lumbotomy.
\end{abstract}

Key words: Live donor, kidney transplantation, laparoscopic nephrectomy.

\section{Resumen}

Introducción: El trasplante renal es en la actualidad el tratamiento de elección de la Insuficiencia Renal Crónica Terminal. La Nefrectomía Laparoscópica del Donante Vivo (NLDV) tiene ventajas sustanciales en relación a la Nefrectomía Clásica. Entre estas se destacan una menor estancia hospitalaria, pronto regreso a la actividad laboral, disminución del dolor post-operatorio, mejores resultados cosméticos, menor pérdida de volumen sanguíneo y una disminución de infecciones de heridas operatorias, consolidándose como la primera prioridad como forma de obtener un órgano para trasplante renal. El presente trabajo tiene como objetivo

*Recibido el 9 de enero de 2014 y aceptado para publicación el 19 de mayo de 2014.

Sin apoyo financiero ni conflictos de interés.

Correspondencia: Dr. Jorge Aguiló M.

jorge_aguilo@hotmail.com 
mostrar la casuística y complicaciones en el Hospital Barros Luco-Trudeau (HBLT), como centro pionero en NLDV en nuestro país. Material y Método: Estudio retrospectivo de corte transversal. Se realiza una revisión de registros clínicos de 75 NLDV realizadas entre 1998-2013, seleccionando datos demográficos, clínicos y quirúrgicos de donantes y receptores, con un especial énfasis en relación a complicaciones perioperatorias. Resultados: Sin mortalidad. Todos los riñones fueron implantados satisfactoriamente en sus respectivos receptores. Cirugía con duración promedio de $116 \mathrm{~min}$, estadía hospitalaria promedio de 1,6 días, isquemia caliente promedio de $6,8 \mathrm{~min}$ e isquemia fría promedio de $31,5 \mathrm{~min}$. Incidentes operatorios $8 \%$ y $4 \%$ conducentes a conversión. Tasa de reoperación de 1,3\%. En cuanto a receptores, un 1,5\% desarrolla Necrosis Tubular Aguda. 2,2\% requiere transplantectomía. Discusión: La NLDV representa una técnica segura, que ha llegado a constituir el 100\% de las nefrectomías de donantes vivos realizadas durante los últimos tres años. Los resultados son satisfactorios y plenamente comparables a los resultados obtenidos por lumbotomía clásica y de otros centros de alto volumen laparoscópico a nivel internacional.

Palabras clave: Trasplante renal, nefrectomía laparoscópica, donante vivo.

\section{Introducción}

El trasplante renal es en la actualidad el tratamiento de elección de la Insuficiencia Renal Crónica Terminal (ICRT). Por sus mejores resultados y la creciente lista de espera por un donante fallecido, el donante vivo es la primera prioridad como forma de obtener un órgano para trasplante. El primer trasplante de donante vivo exitoso se realizó hace más de 50 años. Desde entonces, el trasplante de donante vivo se ha convertido en el mejor tratamiento en este tipo de patología ${ }^{1-3}$.

Ratner realizó la primera Nefrectomía Laparoscópica de Donante Vivo (NLDV) en 1995, como alternativa a la Nefrectomía Clásica (NC). Desde aquel hito histórico, numerosos centros quirúrgicos se han entrenado en este procedimiento. En este contexto se realiza en Chile en 1998 el primer caso en el Hospital Barros Luco Trudeau ${ }^{4}$, posteriormente extendiéndose a otros centros quirúrgicos del país.

La NLDV tiene ventajas sustanciales en relación a la NC, destacando una menor estancia hospitalaria, un pronto regreso a la actividad laboral, una disminución del dolor post-operatorio, mejores resultados cosméticos, menor pérdida de volumen sanguíneo y una disminución de infecciones de heridas operatorias, entre otras ${ }^{5,6}$. Estas ventajas han sido aprovechadas para promover un aumento en la donación renal. En Estados Unidos, este tipo de procedimiento alcanza el 50\% del total de las nefrectomías en donantes vivos y dicho porcentaje ha ido en rápido aumento.

A pesar de todos los beneficios de la NLDV, esta no está exenta de complicaciones, las que adquieren una especial relevancia considerando su carácter electivo y altruista.

En cuanto al resultado del trasplante, se señala al comparar en un análisis de la base de datos de la UNOS a 2.734 pacientes nefrectomizados por vía laparoscópica con 2.576 pacientes trasplantados por lumbotomía clásica, que no hubo diferencia significativa en el resultado en ambos tipos de nefrectomía en el mediano plazo.

\section{Material y Método}

Estudio retrospectivo de corte transversal.

Se realiza una revisión de resultados de las 75 NLDV realizadas en el HBLT entre el año 1998 y marzo de 2013, seleccionando y analizando datos demográficos, clínicos y quirúrgicos, con un especial énfasis en las complicaciones peri-operatorias.

Se trabajan datos estadísticos utilizando planilla de cálculos Microsoft Excel $2011^{\circledR}$.

La técnica quirúrgica utilizada en los casos corresponde a la misma utilizada por Ratner y Flowers $^{2}$, con leves modificaciones.

Los primeros 20 casos fueron realizados con stappler vascular y la extracción renal se realizó mediante incisión umbilical y los siguientes 55 casos se utilizó Hemolock para el control vascular. La técnica utilizada en la actualidad tiene algunas modificaciones y se describe a continuación:

Posición de lumbotomía, se utilizan 4 trocares para el lado izquierdo y 5 para el lado derecho. En caso de nefrectomía izquierda, utilizando bisturí armónico se procede a un desprendimiento completo de colon desde el parietocólico, sobrepasando el nivel de la aorta. La disección vascular comienza con la vena renal, hasta llegar a la vena cava. Para el control vascular utilizamos doble Hemolock a la vena y arteria renal, seccionando sus colaterales gonadales y suprarrenales. La arteria o arterias renales se disecan hasta su nacimiento en la aorta. En caso de nefrectomía derecha se realiza un procedimiento similar, bajando duodeno y colon derecho. El uréter es disecado junto a la vena gonadal y luego se secciona en el cruce con la arteria ilíaca. Se extrae el riñón con toda la grasa perirrenal a través de una incisión tipo McBurney en fosa ilíaca ${ }^{4}$. 
Tabla 1. Tasa de incidentes operatorios y conversiones clasificadas según Satava

\begin{tabular}{|lcc|}
\hline Incidentes operatorios & Conversión & n: 6(8\%) \\
Sangrado venoso unión vena espermática & Sí & 3 \\
Sangrado arteria renal por stappler disfuncional & Sí & $(4 \%)$ \\
Desprendimiento clip vena renal una vez seccionada & Sí & 3 \\
Sección de 2 ramas provenientes de arteria renal & No & $(4 \%)$ \\
Desgarro de vena renal & No \\
Uréter seccionado proximal & No \\
\hline
\end{tabular}

\section{Resultados}

Se obtienen datos de 75 pacientes: 37 hombres $(49,3 \%)$ y 38 mujeres $(50,6 \%)$ de entre 27 y 60 años, con un promedio de 41,2 años de edad.

Respecto al sitio quirúrgico se realizaron 71 nefrectomías izquierdas $(94,7 \%)$ y 4 nefrectomías derechas $(5,3 \%)$.

La cirugía tuvo una duración promedio de 116 min, con un rango de 75-175 min y la estadía hospitalaria promedio fue de 1,6 días, con un rango de entre 2-25 días.

La isquemia caliente fue en promedio de $6,8 \mathrm{~min}$, con un rango de 5-15 min, mientras que el promedio isquemia fría fue de $31,5 \mathrm{~min}$, con un rango de 11$120 \mathrm{~min}$.

Las tasas de incidentes operatorios y conversiones fueron clasificadas según Satava, se encuentran descritas en la Tabla 1.

Se aprecian 6 incidentes intraoperatorios $(8 \%)$. De ellos 3 son conducentes a conversión (4\%) y 3 son resueltos satisfactoriamente vía laparoscópica (4\%). De los casos conducentes a conversión, hubo un sangrado venoso de la unión de la vena espermática, un sangrado de la arteria renal por un stappler disfuncional y un desprendimiento de clip de la vena renal una vez seccionada. De los casos no conducentes a conversión se describe una sección de 2 ramas provenientes de la arteria renal, un desgarro de la vena renal y un uréter seccionado proximal, que en el implante fue anastomosado al uréter propio.

Respecto a las complicaciones post-operatorias hubo una complicación post-operatoria mediata en la serie, correspondiendo a un 1,3\% del total. El caso trata de una paciente quien cursa con hemoperitoneo masivo por movilización de Hemolock que debió ser re-operada al primer día post-operatorio (Clavien IV o complicación con riesgo vital que requiere cama crítica en su post-operatorio). Este caso explica el aumento de rango en la estadía hospitalaria en la serie ( 25 días).

En el período post-operatorio alejado (3 meses) un paciente presenta hernia incisional en brecha de fosa ilíaca izquierda (Clavien I o desviación del curso quirúrgico normal sin necesidad de manejo quirúrgico o farmacológico inmediato).
En cuanto a incidentes en receptores, en la serie se describen 2 casos de NTA (1,5\%), que requirieron de hemodiálisis post-operatoria y 3 casos conducentes a pérdida del injerto $(2,2 \%)$ : una trombosis vascular idiopática, una trombosis vascular por anomalía anatómica importante (pelvis extrarrenal + 2 arterias renales + hilio renal lateral) y un rechazo del injerto a los 30 días.

La creatinina de receptores a la semana fue de 1,81 con un rango de 0,98 a 11,55 y al mes fue de 1,37 , con un rango de 0,71 a 8,4 .

La función renal post-operatoria de los donantes renales vivos ha sido históricamente satisfactoria y ampliamente reportada en la literatura ${ }^{7}$. Por este motivo no es estudiada en forma rutinaria durante el seguimiento.

Todos los riñones fueron implantados satisfactoriamente en sus respectivos receptores. En la serie no hubo mortalidad.

\section{Discusión}

En el transcurso del presente trabajo se han expuesto los principales resultados clínicos y quirúrgicos de la NLDV y las complicaciones perioperatorias reportadas. También hemos incluido una descripción detallada de la técnica quirúrgica empleada por nuestro centro.

Relativo al sitio quirúrgico y al igual que otros grupos, el riñón de elección para trasplante renal es el izquierdo. Lo anterior se fundamenta en una vena renal de menor longitud y en sus relaciones anatómicas. El riñón derecho sólo debe ser utilizado cuando hay una ventaja significativa para el paciente.

Con respecto a los tiempos de isquemia podemos mencionar que la isquemia caliente históricamente ha sido mayor en la técnica laparoscópica, que en la técnica abierta ${ }^{8-10}$. A pesar de lo anterior, tiempos menores a 17 min no se relacionan con efectos deletéreos en la función renal del receptor ${ }^{8,9}$. En este aspecto $\mathrm{y}$, a pesar de que esta debiera intentar ser mínima, el promedio de isquemia caliente de nuestra serie es menor al tope máximo seguro.

$\mathrm{Al}$ analizar tiempos de isquemia fría, se observan casos con tiempos prolongados de hasta $120 \mathrm{~min}$. 
Estos corresponden a nefrectomías cuyo receptor se encuentra geográficamente distante, como es el caso de pacientes trasplantados en el Hospital de niños Exequiel González Cortés.

En relación a las complicaciones intra-operatorias, y complicaciones postoperatorias, éstas son reportadas en la literatura con una frecuencia de $6,8 \%$ y $17,1 \%{ }^{11}$ de los casos, respectivamente. En nuestra serie se observa una tasa similar $(8 \%)$, sin presencia de lesiones intestinales, pleurales, esplénicas, hepáticas, diafragmáticas o ureterales observadas en otros grupos de trabajo ${ }^{8-10}$.

El caso asociado a gran morbilidad consistió en un desplazamiento de hemolock, que provocó una hemorragia masiva al primer día post-operatorio. Este caso permaneció hospitalizado por 25 días, siendo el resto de los pacientes dados de alta al primer día post-operatorio. Posterior a dicho caso se modificó la técnica utilizando doble hemolock para lograr control vascular arterial.

La tasa de conversión (4\%) demostró ser ligeramente mayor a lo observado en la literatura. Esto se ha explicado por la curva de aprendizaje realizada por el equipo quirúrgico. Al día de hoy, desde el año 2010 que no se realiza una nefrectomía por lumbotomía.

Se ha observado una disminución mantenida en la tasa de complicaciones a medida que los centros obtienen y consolidan equipos, ganan experiencia y avanzan en la curva de aprendizaje. Para disminuir al mínimo los riesgos asociados a la cirugía su práctica debería restringirse a centros de alta frecuencia ${ }^{11}$.

Con todo lo anterior en consideración, el tiempo quirúrgico obtenido en el HBLT es menor al observado en otras series ${ }^{1,6}$.

Respecto a la mortalidad, Kasiske y cols., han estimado la mortalidad en 3 muertes por cada 10.000 nefrectomías $(0,03 \%)$ y la tasa de reoperación de un 0,5 y $1 \%{ }^{12}$. En nuestra serie no hubo mortalidad y hubo un solo caso asociado a gran morbilidad, lo que corresponde a $1,3 \%$ del total.

Con relación a los valores séricos de creatinina, se ha descrito que la técnica laparoscópica, presenta una recuperación de creatinina más lenta que su contraparte abierta ${ }^{13}$. Los valores de creatinina de la serie medidos a la semana y al mes post-operatorios estuvieron dentro de un rango aceptable y no son mayores a los descritos en la técnica abierta.

En definitiva, la NLDV representa una técnica segura, pionera en nuestro país, que ha llegado a constituir el $100 \%$ de las nefrectomías de donantes vivos realizadas en nuestro hospital durante los últimos tres años (2010-2013). Los resultados son satisfactorios y plenamente comparables a los resultados obtenidos por lumbotomía clásica ${ }^{14}$ y de otros centros de alto volumen laparoscópico a nivel internacional.

\section{Referencias}

1. Shapiro R, Brennan DC. Benefits and complications of laparoscopic donor nephrectomy. In UpToDate, Post TW (Ed), UpToDate, Waltham, M.A (Accessed on May 18, 2014)

2. Ratner LE, Ciseck LJ, Moore RG, Francisco GC, Howard SK, Kavoussi LR. Laparoscopic live donor nephrectomy. Trasplantation 1995;60:1047-9.

3. Flowers JL, Jacobs S, Cho E, Morton A, Rosenberger WF, Evans D, et al. Comparison of open and laparoscopic live donor nephrectomy. Ann Surg. 1997;226:483-90.

4. Aguiló J, Vergara J. Nefrectomía laparoscópica en donante vivo versus cirugía abierta y mano-asistida. II Curso de Trasplantes Año 2002, Sociedad Chilena de Trasplante, Santiago, noviembre de 2003.

5. Alcaraz A, Rosales A, Palou J, Caffaratti J, Montlleó M, Segarra J, y cols. Nefrectomía laparoscópica de donante vivo para trasplante renal. Los dos primeros años de experiencia. Arch Esp Urol. 2004;57:1091-8.

6. Rodríguez O, Breda A, Esquena S, Villavicencio H. Aspectos quirúrgicos actuales de la nefrectomía de donante vivo. Actas Urol Esp. 2012;37:181-7.

7. Goldfarb DA, Matin SF, Braun WE, Schreiber MJ, Mastroianni BD, Papajcik BD, et al. Renal outcome 25 years after donor nephrectomy. J Urol. 2001;166:2043-7.

8. Nanidis TG, Antcliffe D, Kokkinos C, Borysiewicz CA, Darzi AW, Tekkis PP, et al. Laparoscopic versus open live donor nephrectomy in renal transplantation: a meta-analysis. Ann Surg. 2008;247:58-70.

9. Wilson C, Sanni A, Rix D, Soomro N. Nefrectomía laparoscópica versus abierta para los donantes de riñón vivos. Cochrane Database of Systematic Reviews 2011 Issue 11. Art. No: CD006124. DOI: 10.1002/14651858. CD006124.

10. Ellison MD, McBride MA, Edwards LB, Taranto SE, Barr ML, Trotter JF, et al. Living organ donation: mortality and early complications among 16.395 living donors in the U.S. Am J Trasplant. 2003;3:283.

11. Jacobs SC, Cho E, Foster C, Liao P, Bartlett ST. Laparoscopic donor nephrectomy: the University of Maryland 6-year experience. J Urol. 2004;171:47-51.

12. Oppenheimer F. Seguimiento del donante vivo a corto, medio y largo plazo. Nefrología 2010;30 (Suppl. 2):1005.

13. Gaete J. Nefrectomía en donante renal vivo: experiencia en 150 casos. Rev Chil Cir. 1989;41:104-9.

14. Linares Quevedo AI, Burgos Revilla FJ, Villafruela Sanz JJ, Zamora Romero J, Pascual Santos J, Marcén Letosa R, y cols. Análisis comparativo de la función del injerto renal de donante vivo tras nefrectomía abierta y laparoscópica: modelo experimental. Actas Urol Esp. 2008;32:140-51. 